\title{
Lung neuroendocrine tumors: a new addition to the evolving list of spread through air spaces
}

\author{
Hironori Uruga ${ }^{1,2}$, Mari Mino-Kenudson ${ }^{3}$ \\ ${ }^{1}$ Department of Respiratory Medicine, Respiratory Center, Toranomon Hospital, Tokyo, Japan; ${ }^{2}$ Department of Respiratory Medicine, Toranomon \\ Hospital Kajigaya, Kawasaki, Japan; ${ }^{3}$ Department of Pathology, Massachusetts General Hospital and Harvard Medical School, Boston, MA, USA \\ Correspondence to: Mari Mino-Kenudson, MD. Department of Pathology, Massachusetts General Hospital, 55 Fruit Street, Warren 122, Boston, MA \\ 02114, USA. Email: mminokenudson@partners.org. \\ Provenance: This is an invited article commissioned by the Academic Editor Dr. Chenyang Dai (Department of Thoracic Surgery, Shanghai \\ Pulmonary Hospital, School of Medicine, Tongji University, Shanghai, China). \\ Comment on: Aly RG, Rekhtman N, Li X, et al. Spread Through Air Spaces (STAS) Is Prognostic in Atypical Carcinoid, Large Cell Neuroendocrine \\ Carcinoma, and Small Cell Carcinoma of the Lung. J Thorac Oncol 2019;14:1583-93.
}

Submitted Aug 21, 2019. Accepted for publication Aug 30, 2019.

doi: $10.21037 /$ tlcr.2019.09.03

View this article at: http://dx.doi.org/10.21037/tlcr.2019.09.03

Air-filled spaces are a notable characteristic of the lung. When a cancer develops in the lung, it is often surrounded by air spaces; thus, there is the possibility that cancer cells spread thorough air spaces. In fact, Kodama and colleagues presented a lung cancer case with multifocal "aerogenous" spread in 1980 and ultrastructurally studied the nature of detached fragments (1). Since then, multiple groups have reported the aerogenous spread of lung cancer cells, but it was not until 2012 when systematic pathological and prognostic analyses of the aerogenous spread of lung cancer cells began conducted. Onozato and colleagues found unique island-like structures in two of four lung adenocarcinomas analysed by $3 \mathrm{D}$ reconstruction and named them as tumor islands $(2,3)$. "Tumor islands" are detached, large clusters of tumor cells in alveolar spaces separated from the main tumor mass but are connected to the main tumor mass in different tissue levels (2). Subsequently, they showed the association of tumor islands with shorter recurrence-free survival in stage I-II lung adenocarcinomas and hypothesized that tumor islands represent 'air space invasion" (3). Kadota and colleagues demonstrated that the spread of single tumor cells or micropapillary/solid type tumor cell clusters within air spaces adjacent to the main tumor [i.e., tumor spread through air spaces (STAS)] is a significant risk factor for shorter recurrence-free survival in patients with small lung adenocarcinomas $(\leq 2 \mathrm{~cm})$ treated with sublobar resection (4). Of note, there appear to be mutual morphologic features between the solid type STAS, in particular, large clusters and tumor islands. Based on these results, STAS was recognized as a new form of invasion, "air space invasion," in the 2015 World Health Organization (WHO) classification and became an exclusion criterion for minimally invasive adenocarcinoma (5). Subsequently, Dai et al. showed no statistical difference in recurrence free survival between patients with stage IA lung adenocarcinoma harboring STAS and those with stage IB suggesting that STAS could be a staging factor (6). In addition, STAS has been seen not only in lung adenocarcinomas but also in squamous cell carcinomas (7-9) and pleomorphic carcinomas (10). Many subsequent studies reported STAS as a predictor of worse patient outcomes, and a systematic review and meta-analysis of 2,897 patients with non-small cell lung carcinoma showed that the presence of STAS was associated with shorter recurrencefree survival [hazard ratio (HR): 1.975; $\mathrm{P}<0.001$ ] (11). More recently, Eguchi and colleagues conducted a propensity score matching analysis on lobectomy $v s$. sublobar resection for stage I lung adenocarcinoma and found shorter recurrence-free and overall survivals of the patients with STAS compared with those without in the sublobar resection cohort, while STAS had no bearing on patients' outcomes in the lobectomy cohort. In addition, the worse outcomes were irrespective of surgical margin-totumor diameter ratio in the STAS+ patients. Accordingly, 
they emphasized the importance of lobectomy for T1N0M0 lung adenocarcinomas with STAS opposed to sublobar resection (12). Thus, when STAS is identified in a sublobar resection specimen, even for a small node negative tumor, completion lobectomy may need to be considered. Interestingly, however, STAS has been shown to be predictor of worse outcomes in patients who underwent lobectomy for other types of lung cancer $(7,8)$.

Although few studies have evaluated STAS and its biological significance in patients with small cell carcinoma (SCLC) and atypical carcinoid (AC) $(13,14)$, no study has investigated the prognostic significance of STAS in a broad spectrum of lung neuroendocrine tumors (NETs) in a comprehensive manner. Given this background, Aly and colleagues examined a prognostic impact of STAS in lung NETs in a study involving 487 surgically resected stage I, II, and III tumors comprising 299 typical carcinoid (TC), 38 AC, 93 large cell neuroendocrine carcinoma (LCNEC), and 57 SCLC (15). In the study, STAS was defined as the presence of more than one floating tumor cell cluster at least one air space away from the tumor boundary, and continuous alveolar space spread from the tumor edge to the furthest STAS was also required. STAS was present in 129 of the 587 patients (22\%), and, in univariable analysis, was associated with higher smoking pack-years, adjuvant chemotherapy, higher pathological stage, presence of lymphatic and vascular invasion, presence of necrosis, higher mitotic count, Ki-67 labeling index, and histologic subtype. The authors also analyzed the incidence and pattern of recurrence and showed a postoperative 5-year recurrence rate of $54 \%$ in patients with STAS and that of $24 \%$ in those without $(\mathrm{P}<0.001)$. Lung cancer-specific cumulative incidence of death (LC-CID) was also higher in patients with STAS than in those without $(43 \% v s .18 \%$, $\mathrm{P}<0.001)$. Of note, the prognostic analyses were performed in only AC, LCNEC, and SCLC $(n=188)$ due to the low recurrence rate in patients with TC. Upon multivariable regression analysis, STAS was associated with a higher recurrence rate $(\mathrm{HR}: 2.85 ; \mathrm{P}<0.001)$ and higher LC-CID (HR: $2.72 ; \mathrm{P}<0.001)$ irrespective of histologic subtypes. As for the pattern of recurrence, locoregional recurrence rates were not significantly different between patients with STAS and those without $(\mathrm{P}=0.8)$, while a distant recurrence rate was higher in patients with STAS $(\mathrm{P}=0.006)$. Further, the patients with STAS had a risk of brain metastasis three times greater than those without $(18 \%$ vs. $5 \%, \mathrm{P}=0.010)$, and lymph node and adrenal metastases were also more prevalent in patients with STAS than in those without
$(\mathrm{P}=0.032$ and $\mathrm{P}=0.020$, respectively). In a subgroup analysis of patients with STAS, a higher incidence of brain metastasis was found in the LCNEC cohort $(23 \% v s$. $6 \%, \mathrm{P}=0.035)$ but not in the SCLC cohort (19\% vs. $7 \%$, $\mathrm{P}=0.195)$.

The study by Aly and colleagues revealed some interesting findings (15). First, STAS was found to be a predictor of worse prognosis in lung AC, LCNEC and SCLC as well as in lung adenocarcinomas, squamous cell carcinomas, and pleomorphic carcinomas. Second, as for the site of recurrence, the incidence of brain metastasis was three times higher in patients with STAS than in those without, while, in the subclass analysis, STAS was a risk factor for brain metastasis only in the LCNEC cohort but not in the SCLC cohort. In previous studies, brain metastasis was found to be very frequent in lung LCNEC and SCLC, occurring in approximately $50 \%$ of cases (16), and prophylactic cranial irradiation (PCI) was able to reduce the risk in half in patients with SCLC, thus leading to the recommendation that the patient with limited-stage SCLC undergo PCI after a standard first-line chemotherapy (17). In the study by Aly and colleagues, 21\% of SCLC and none of LCNEC patients received PCI (15), and this might have contributed to the difference in the brain recurrence rate between LCNEC and SCLC in the STAS+ group. Thus, it is reasonable to think that PCI may be beneficial for patients who are found to have LCNEC harboring STAS in lung resection as well as those with SCLC. The third interesting finding is that the recurrence pattern was different between adenocarcinoma and NETs when the tumor exhibits STAS. Kadota $e t a l$. originally reported that STAS was associated with increased risks of locoregional and distant recurrence in patients with small stage I lung adenocarcinoma who had undergone sublobar resection, but not in those who had undergone lobectomy (4). Aly et al. recently performed 3-dimensinal reconstruction of STAS in lung TC and AC, and have demonstrated a connection between STAS and the main tumor similar to tumor islands (18). These data may indicate some biological differences of STAS between adenocarcinoma and NE tumors or other types of lung cancer. In adenocarcinoma, single tumor cells, micropapillary and/or small clusters of tumor cells may travel far from the main tumor, and if tumor cells remain the remnant lung parenchyma after sublobar resection, they may become a nidus of tumor recurrence (19). In contrast, STAS in the other types of lung tumor including NETs that exhibits solid clusters (similar to tumor islands) may merely reflect the aggressive biology/invasive capability of the tumor. 
Although multiple studies have contributed to improving our understanding of STAS and its biological significance, there remain several questions. The fundamental question is whether STAS is an in vivo phenomenon or an artifact, and this issue has long been under an intensive discussion. The phenomenon of floating tumor cells in alveolar spaces created artificially during processing at the pathology laboratory was named "spreading through a knife surface" (STAKS) by Thunnissen et al. (20). They have demonstrated in a multicenter study that the number of free tumor cell clusters within air spaces is increased by knife cuts, and $93 \%$ of the tumor cell clusters could be explained by mechanical forces associated with tissue handling (21). Many studies encompassing various histological types, however, have reproducibly shown STAS to be a risk factor for postoperative recurrence (4,6-11). Furthermore, as already discussed, large solid clusters of STAS/tumor islands have been proven to be a direct extension from the main tumor. Thus, it is reasonable to think that at least a part of STAS is real, and STAS in general reflects aggressive tumor biology irrespective of how it develops or is generated.

Nevertheless, it is important to confidently differentiate STAS from "true" artifacts (to prevent potentially unnecessary completion lobectomy in patients with adenocarcinoma). Unfortunately, however, the criteria for STAS have not been standardized yet and high concordance on the diagnosis of STAS by pathologists needs to be achieved. The multiple studies have employed various definitions of STAS including cut-offs for a number of tumor clusters/cells and/or distance from the main tumor (22). For instance, Morimoto and colleagues defined STAS as more than 3 clusters containing $<20$ nonintegrated micropapillary tumor cells located $>3 \mathrm{~mm}$ away from the main tumor to differentiate STAS from artifacts (23). In the current study (15), Aly and colleagues described several features of artifacts as exclusion criteria of STAS: (I) mechanically dissociated tumor floaters (clusters of tumor cells with a ragged-edge, located randomly and/or located at the edge of the tumor section); (II) normal benign pneumocytes or bronchial cells; (III) strips of tumor cells detached from alveolar walls or stroma due to poor preservation; (IV) isolated tumor clusters distantly situated away from the tumor not in a continuous manner. They also considered the presence of a single focus of STAS in the entire tumor as an artifact. In another study by Uruga and colleagues, a semiquantitative assessment of STAS in patients with resected adenocarcinoma showed that high STAS ( $\geq 5$ clusters of STAS in one $200 \times$ microscopic field) was associated with shorter recurrence-free survival compared to no STAS, but that low STAS (1-4 clusters of STAS in one 200x microscopic field) was not (24). Thus, while 5 or more clusters of STAS may be relevant in terms of survival, the significance of a few (2-4) clusters of STAS remains uncertain. As for the reproducibility of STAS among pathologists, in the study by Monroig-Bosque and colleagues, the diagnosis of STAS in 30 adenocarcinomas with significant micropapillary pattern varied from $34.1 \%$ to $52.8 \%$ (mean $43.4 \%$ ) among 15 pulmonary pathologists (25). Further large-scale studies are warranted to elucidate and improve the reproducibility of the STAS diagnosis by pathologists.

In summary, Aly et al. (15) found STAS to be a poor prognostic factor in lung NETs (AC, LCNEC and SCLC). Furthermore, brain metastasis occurred more frequently in patients with STAS than in those without. These results might be useful for the postoperative management of patients with lung NET. Although our understanding of STAS and its prognostic significance in lung cancer has significantly advanced in the last few years, there remain multiple questions about STAS. Thus, further studies are warranted to address these questions.

\section{Acknowledgments}

None.

\section{Footnote}

Conflicts of Interest: M Mino-Kenudson serves as a consultant for Merrimack Pharmaceuticals and H3 Biomedicine. $\mathrm{H}$ Uruga has no conflicts of interest to declare.

Ethical Statement: The authors are accountable for all aspects of the work in ensuring that questions related to the accuracy or integrity of any part of the work are appropriately investigated and resolved.

\section{References}

1. Kodama T, Kameya T, Shimosato Y, et al. Cell incohesiveness and pattern of extension in a rare case of bronchioloalveolar carcinoma. Ultrastruct Pathol 1980;1:177-88.

2. Onozato ML, Klepeis VE, Yagi Y, et al. A role of threedimensional (3D)-reconstruction in the classification of lung adenocarcinoma. Anal Cell Pathol (Amst) 2012;35:79-84.

3. Onozato ML, Kovach AE, Yeap BY, et al. Tumor islands in 
resected early-stage lung adenocarcinomas are associated with unique clinicopathologic and molecular characteristics and worse prognosis. Am J Surg Pathol 2013;37:287-94.

4. Kadota K, Nitadori J, Sima CS, et al. Tumor Spread through Air Spaces is an Important Pattern of Invasion and Impacts the Frequency and Location of Recurrences after Limited Resection for Small Stage I Lung Adenocarcinomas. J Thorac Oncol 2015;10:806-14.

5. Travis WD, Brambilla E, Burke AP, et al. WHO Classification of Tumours of the Lung, Pleura, Thymus and Heart. 4th Edition. Lyon, France: World Health Organization, 2015.

6. Dai C, Xie H, Su H, et al. Tumor Spread through Air Spaces Affects the Recurrence and Overall Survival in Patients with Lung Adenocarcinoma $>2$ to $3 \mathrm{~cm}$. J Thorac Oncol 2017;12:1052-60.

7. Kadota K, Kushida Y, Katsuki N, et al. Tumor Spread Through Air Spaces Is an Independent Predictor of Recurrence-free Survival in Patients With Resected Lung Squamous Cell Carcinoma. Am J Surg Pathol 2017;41:1077-86.

8. Lu S, Tan KS, Kadota K, et al. Spread through Air Spaces (STAS) Is an Independent Predictor of Recurrence and Lung Cancer-Specific Death in Squamous Cell Carcinoma. J Thorac Oncol 2017;12:223-34.

9. Yanagawa N, Shiono S, Endo M, et al. Tumor spread through air spaces is a useful predictor of recurrence and prognosis in stage I lung squamous cell carcinoma, but not in stage II and III. Lung Cancer 2018;120:14-21.

10. Yokoyama S, Murakami T, Tao H, et al. Tumor Spread Through Air Spaces Identifies a Distinct Subgroup With Poor Prognosis in Surgically Resected Lung Pleomorphic Carcinoma. Chest 2018;154:838-47.

11. Chen D, Mao Y, Wen J, et al. Tumor Spread Through Air Spaces in Non-Small Cell Lung Cancer: a systematic review and meta-analysis. Ann Thorac Surg 2019;108:945-54.

12. Eguchi T, Kameda K, Lu S, et al. Lobectomy Is Associated with Better Outcomes than Sublobar Resection in Spread through Air Spaces (STAS)-Positive T1 Lung Adenocarcinoma: A Propensity Score-Matched Analysis. J Thorac Oncol 2019;14:87-98.

13. Toyokawa G, Yamada Y, Tagawa T, et al. High Frequency of Spread Through Air Spaces in Resected Small Cell Lung Cancer. Anticancer Res 2018;38:1821-5.

14. Altinay S, Metovic J, Massa F, et al. Spread through air spaces (STAS) is a predictor of poor outcome in atypical carcinoids of the lung. Virchows Arch 2019;475:325-34.

15. Aly RG, Rekhtman N, Li X, et al. Spread Through Air Spaces (STAS) Is Prognostic in Atypical Carcinoid,
Large Cell Neuroendocrine Carcinoma, and Small Cell Carcinoma of the Lung. J Thorac Oncol 2019;14:1583-93.

16. Metro G, Ricciuti B, Chiari R, et al. Survival outcomes and incidence of brain recurrence in high-grade neuroendocrine carcinomas of the lung: Implications for clinical practice. Lung Cancer 2016;95:82-7.

17. Aupérin A, Arriagada R, Pignon JP, et al. Prophylactic cranial irradiation for patients with small-cell lung cancer in complete remission. Prophylactic Cranial Irradiation Overview Collaborative Group. N Engl J Med 1999;341:476-84.

18. Aly R, Teplov A, Uraoka N, et al. Whole Block Imaging (WBI) by MicroCT: of Lung Carcinoid Tumors:

3-Dimensinal Morphometric Analysis of Spread Through Air Spaces (STAS). United States \& Canadian Academy of Pathology 108th Annual Meeting. 2019.

19. Dai C, Xie H, Su H, et al. Tumor spread through air spaces (STAS): a call for more evidence. J Thorac Dis 2017;9:E951-3.

20. Thunnissen E, Blaauwgeers HJ, de Cuba EM, et al. Ex Vivo Artifacts and Histopathologic Pitfalls in the Lung. Arch Pathol Lab Med 2016;140:212-20.

21. Blaauwgeers H, Flieder D, Warth A, et al. A Prospective Study of Loose Tissue Fragments in Non-Small Cell Lung Cancer Resection Specimens: An Alternative View to "Spread Through Air Spaces". Am J Surg Pathol 2017;41:1226-30.

22. Warth A. Spread through air spaces (STAS): a comprehensive update. Transl Lung Cancer Res 2017;6:501-7.

23. Morimoto J, Nakajima T, Suzuki H, et al. Impact of free tumor clusters on prognosis after resection of pulmonary adenocarcinoma. J Thorac Cardiovasc Surg 2016;152: 64-72.e1.

24. Uruga H, Fujii T, Fujimori S, et al. Semiquantitative Assessment of Tumor Spread through Air Spaces (STAS) in Early-Stage Lung Adenocarcinomas. J Thorac Oncol 2017;12:1046-51.

25. Monroig-Bosque PDC, Morales-Rosado JA, Roden AC, et al. Micropapillary adenocarcinoma of lung: Morphological criteria and diagnostic reproducibility among pulmonary pathologists. Ann Diagn Pathol 2019;41:43-50.

Cite this article as: Uruga $\mathrm{H}$, Mino-Kenudson $\mathrm{M}$. Lung neuroendocrine tumors: a new addition to the evolving list of spread through air spaces. Transl Lung Cancer Res 2019;8(Suppl 4):S443-S446. doi: 10.21037/tlcr.2019.09.03 